\title{
中空芳香螺旋的合成及其对碱金属离子的选择性研究
}

\author{
李文芳朱俊彦董泽元* \\ (吉林大学化学学院 超分子结构与材料国家重点实验室 长春 130012)
}

\begin{abstract}
摘要 以非常简便的方法合成了一类新型的中空芳香螺旋, 通过紫外光谱分析发现, 此类中空螺旋结构对碱金属离子 具有选择性识别作用, 并确定了其选择顺序为 $\mathrm{Rb}^{+}>\mathrm{K}^{+}>\mathrm{Cs}^{+}>\mathrm{Na}^{+}>\mathrm{Li}^{+}$. 此外, 研究了芳香螺旋 $\mathbf{A H}$ 对结合最强的 $\mathrm{Rb}$ 离子的苂光滴定分析，进一步证实了这类螺旋中空结构对阳离子的通道内识别作用，以及对碱金属离子具有灵敏的 响应性.
\end{abstract}

关键词＼cjkstart芳香蝶旋; 中空结构; 碱金属离子; 选择性识别

\section{Synthesis of Hollow Aromatic Helix and Their Selective Recognition for Alkali Metal lons}

\author{
Li, Wenfang Zhu, Junyan Dong, Zeyuan* \\ (State Key Laboratory of Supramolecular Structure and Materials, College of Chemistry, Jilin University, Changchun 130012)
}

\begin{abstract}
A novel type of hollow aromatic helices was prepared according to an improved organic synthesis approach. By means of UV-Vis spectroscopy, the recognition and selectivity of aromatic helix (AH) for alkali metal ions were investigated. The results indicated that $\mathbf{A H}$ could specifically recognize the alkali metal ions with an order of $\mathrm{Rb}^{+}>\mathrm{K}^{+}>\mathrm{Cs}^{+}>\mathrm{Na}^{+}>\mathrm{Li}^{+}$. In addition, the fluorescence titration was carried out by adding $\mathrm{Rb}^{+}$into the $\mathbf{A H}$ solution, which further underpinned the binding capacity and sensitive responsiveness of $\mathbf{A H}$ for the alkali metal ions.
\end{abstract}

Keywords aromatic helix; hollow channel; alkali metal ions; selective recognition

天然离子通道是细胞正常活动不可或缺的部分, 细 胞通过它们与周围环境进行着高效且具有选择性的离 子交换 ${ }^{[1]}$. 为了模拟天然离子通道的结构与性质, 科学 家们已经设计合成了大量的离子通道模拟物 ${ }^{[2-5]}$. 鉴于 细胞膜的结构特征, 人造离子通道需要满足通道长度大 约在 $3.5 \mathrm{~nm}$ 的尺度. 利用超分子自组装的方法可以比较 容易地实现人造离子通道的构建. 然而, 由于其非共价 动态超分子结构的特点, 这类人造离子通道存在浓度依 赖性, 并且在膜上的稳定性较差. 这些因素限制了对其 通道性质进行深入研究的可能. 膜蛋白通道的发现让化 学家们尝试去设计制备单分子型的人造离子通道, 然而 其合成制备是目前该领域的重要挑战. 利用大环分子作 为骨架, 希望实现单分子型的人造离子通道的构筑. 但 是, 这类结构可选择的大环模板分子的种类有限, 通道 尺寸很难调节. 近些年, 人造螺旋结构的发展让其成为
理想的离子通道结构模型. 本文合成了具有中空结构的 螺旋寡聚物.

螺旋广泛存在于自然界中, 天然大分子如 DNA、蛋 白质、胶原等都呈现出神奇的螺旋结构 ${ }^{[6]}$, 它们在生物 体系中扮演着非常重要的角色. 借助于丰富的驱动力和 化学结构的多样性, 科学家们已经设计合成了一系列芳

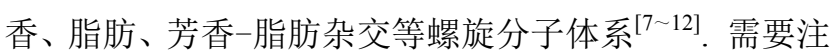
意的是, 稳定性问题极大地限制了大多数螺旋分子体系 的深入研究. 芳香螺旋结构是它们中最稳定的一类, 由 于分子内较强的非共价作用力, 如氢键、静电和 $\pi-\pi$ 相 互作用等, 这类芳香螺旋结构在溶液和固态条件下可以 稳定存在. 因而, 芳香螺旋分子能设计成中空结构, 从 而实现分子的特异性识别 ${ }^{[13]}$. 然而, 制备单分子型的螺 旋离子通道(通道长度大约 $3.5 \mathrm{~nm}$ )面临着巨大的挑战, 主要是由于螺旋合成过程中反应活性的降低以及空间

\footnotetext{
*E-mail: zdong@jlu.edu.cn

Received January 4, 2016; revised May 9, 2016; published online May 17, 2016.

Project supported by the National Natural Science Foundation of China (Nos. 21274051, 21574054).

国家自然科学基金(Nos. 21274051, 21574054)资助项目.
} 
位阻效应的增大. 我们近来改良了螺旋结构的合成方 法, 即先合成尺寸匹配的线性分子, 再通过分子主链的 环化反应形成螺旋结构, 从而实现单分子型的螺旋离子 通道的构建. 本文, 我们将报道螺旋寡聚物的合成以及 其中空结构对离子的识别研究, 为未来深入探索提供研 究基础.

螺旋结构 $\mathbf{A H}$ 是我们近来发现的一类稳定的中空芳 香螺旋. 通过扫描透射显微镜(STM)技术, 我们能够直 接观测到单分子尺寸的螺旋骨架, 这是迄今为止在螺旋 结构表征领域能观察到的直径最小的螺旋. 螺旋结构 AH 由大约 3.2 个重复单元形成一个螺旋周期, 其螺距 为 $0.36 \mathrm{~nm}$, 表明其螺旋结构的形成主要依赖分子内的 $\pi-\pi$ 相互作用. 螺旋结构 $\mathbf{A H}$ 在固态以及溶液中均能稳 定存在. 有趣的是, 在溶液浓度很低的条件下, 螺旋结 构 $\mathbf{A H}$ 是以单螺旋形式存在. 然而, 增加浓度会观察到 AH 从单螺旋到双螺旋的转变过程 ${ }^{[14]}$. 值得注意的是,
螺旋结构 AH 具有一个很显著的特征，即其中空结构. 其结构特点意味着它有潜力发展成为螺旋通道分子(图 1). 因此, 我们研究了具有中空结构的螺旋分子 $\mathbf{A H}$ 对 碱金属离子的选择性，并利用苂光滴定的方法证实了其 中空空腔对 $\mathrm{Rb}^{+}$的选择性偏好. Scheme 1 展示了螺旋结 构 AH 的合成路线，其非常简便的制备方法有助于我们 未来制备理想通道长度的螺旋分子.

\section{1 结果与讨论}

\section{1 芳香螺旋 $\mathrm{AH}$ 的合成}

得到双羧基的化合物 1 后，在充分干燥与氮气保护 条件下, 滴加入肼的四氢呋喃溶液, 在活化剂 PyBOP 存 在下加热反应 $20 \mathrm{~h}$. 相对于高聚螺旋分子, 本文中加热 温度低，反应时间短，获得化合物 2 . 最后将化合物 2 在 三氯氧磷 $\left(\mathrm{POCl}_{3}\right)$ 中 $105{ }^{\circ} \mathrm{C}$ 者沸 $5 \mathrm{~h}$, 此步骤中, 温度维 持在 $105{ }^{\circ} \mathrm{C}$, 适当缩短反应时间可得到螺旋寡聚物. 反

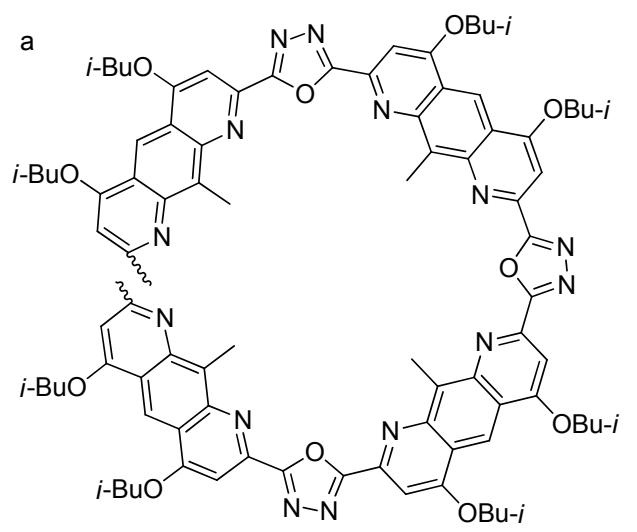
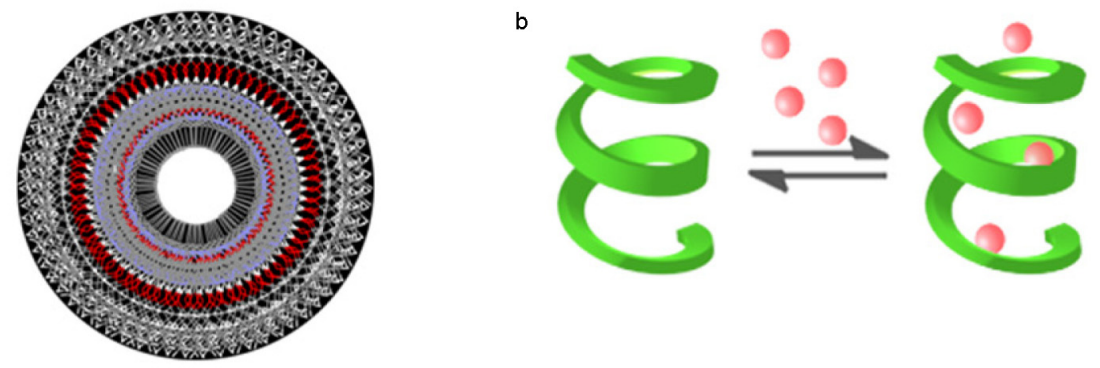

图 1 (a)芳香螺旋分子 $\mathbf{A H}$ 结构及其通道示意图和(b)螺旋中空结构对阳离子的通道内识别作用机理图

Figure 1 (a) Structure of aromatic helix $\mathbf{A H}$ and the channel structure model, (b) the mechanism of identification between the hollow structure belonging to the helix to the cations
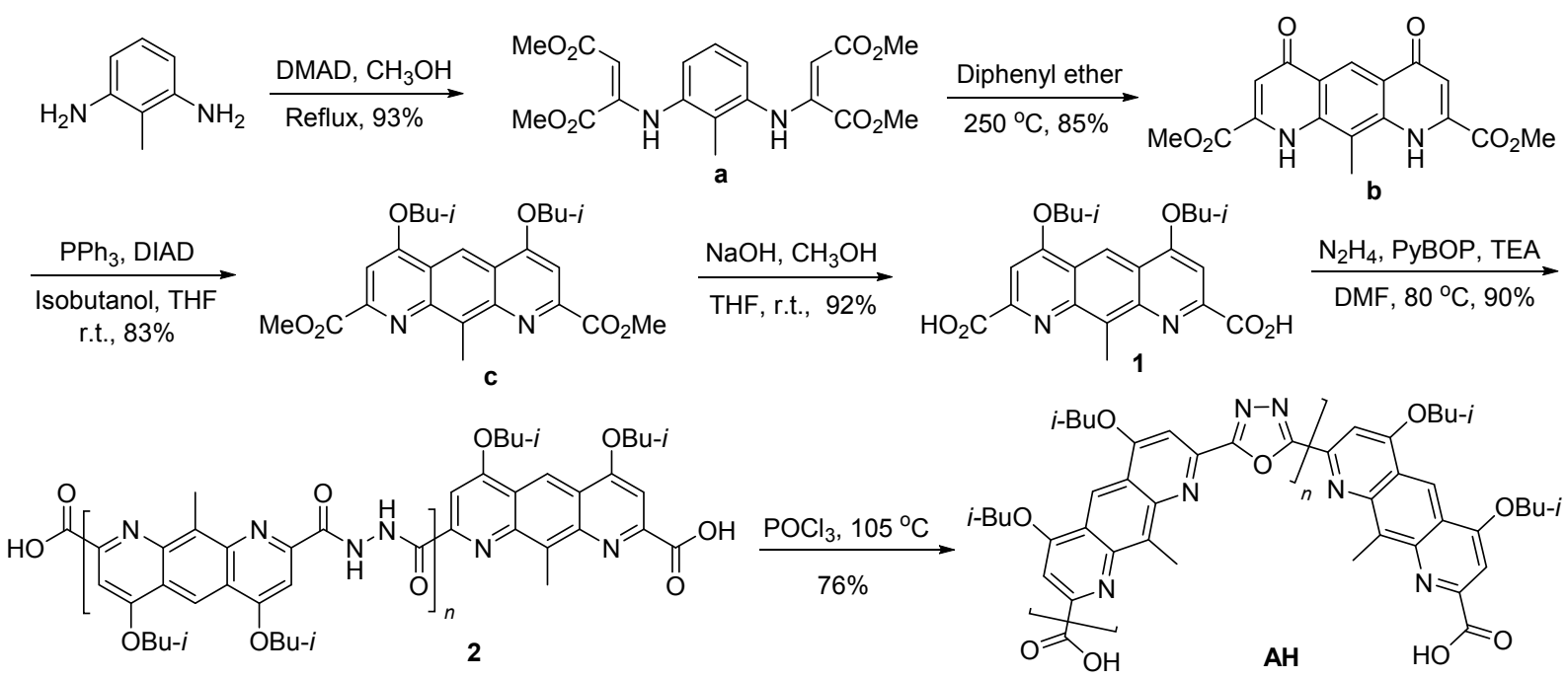

图式 1 芳香螺旋分子 $\mathbf{A H}(n \approx 7)$ 的合成路线

Scheme 1 Synthetic route of aromatic helix AH 
应时间过短(例如小于 $3 \mathrm{~h}$ )则会导致线性分子 2 不能完全 环化. 反应完成后, 经酸碱中和、萃取得到目标产物芳 香螺旋 $\mathbf{A H}^{[14]}$. 通过凝胶渗透色谱 (GPC) 分析证实芳香 螺旋 $\mathbf{A H}$ 的数均分子量为 $2900, \mathrm{PDI}$ 为 1.18 , 主要为七 聚体的寡聚物.

\section{2 芳香螺旋的内部空腔对碱金属离子的选择性}

我们首先进行了芳香螺旋 $\mathbf{A H}$ 对碱金属离子的响应 性测试, 实验发现其对碱金属离子具有明显的响应性和 选择性. 图 2 是在终浓度为 $17 \mu \mathrm{mol} / \mathrm{L}$ 的芳香螺旋 $\mathbf{A H}$ 的 DMSO 溶液中加入终浓度为 $0.35 \mathrm{mmol} / \mathrm{L}$ 的碱金属盐 的水溶液测得的紫外光谱图. 对比芳香螺旋 $\mathbf{A H}$ 本身的 紫外吸收光谱图, 碱金属离子的加入导致其紫外吸收显 著增加, 表明 AH 对碱金属离子具有明显的响应性. 更 重要的发现是, 当加入浓度相同的不同碱金属离子时, 体系中的紫外吸收值呈现不同程度的增加, 这一结果表 明该螺旋内部空腔对碱金属离子具有选择性识别的特 性. 我们确定了其选择性顺序为: $\mathrm{Rb}^{+}>\mathrm{K}^{+}>\mathrm{Cs}^{+}>\mathrm{Na}^{+}$ $>\mathrm{Li}^{+}$. 芳香螺旋 $\mathbf{A H}$ 对碱金属离子的选择性差异主要 归处于其中空空腔尺寸. 正如此类具有空腔的平面大环 结构 ${ }^{[15]}$, 芳香螺旋平面苯环间存在的强 $\pi-\pi$ 作用力使得 螺旋结构非常稳定. 而空腔内的 “ $\mathrm{N}-\mathrm{O}-\mathrm{N}$ ” 原子-金 属匹配位点, 能有效选择识别金属离子. 显然, 由于螺 旋 $\mathbf{A H}$ 的内部结构环境以及其空腔尺寸(大约为 $0.5 \mathrm{~nm}$, 见图 1), 它们对碱金属离子的识别强弱与它们的空间匹 配程度相关. 对尺寸最小的 $\mathrm{Li}^{+}$离子来讲, 螺旋 $\mathbf{A H}$ 的内 部尺寸太大, 而对于尺寸最大的 $\mathrm{Cs}^{+}$离子而言, 其内部 结构中 “ $\mathrm{N}-\mathrm{O}-\mathrm{N}$ ” 三中心结合位点并不能很好地与其 匹配, 因而这类结构对 $\mathrm{Rb}^{+}$表现出了最强的识别能力. 随后, 我们利用荧光光谱技术观察了螺旋 $\mathbf{A H}$ 对 $\mathrm{Rb}^{+}$离 子的响应性(图 3). 将不同浓度的 $\mathrm{Rb}^{+}$离子溶液对同一 浓度 $(17 \mu \mathrm{mol} / \mathrm{L})$ 螺旋 $\mathbf{A H}$ 进行测试, 发现随着 $\mathrm{Rb}^{+}$离子 浓度增加, 苂光强度逐渐增强, 清晰地证实了芳香螺旋 $\mathrm{AH}$ 对碱金属离子 $\mathrm{Rb}^{+}$的识别能力.

\section{2 结论}

本文报道了一种改良的合成方法来制备一类新型 的中空芳香螺旋结构, 解决了芳香螺旋合成过程中反应 活性降低以及空间位阻效应增大导致合成困难的问题. 这类螺旋结构在固态和溶液中具有良好的稳定性, 将有 助于大尺度的螺旋结构的制备与性质研究. 螺旋结构 AH 由大约 3.2 个重复单元形成一个螺旋周期, 其螺距 为 $0.36 \mathrm{~nm}$, 表明其螺旋结构的形成主要依赖分子内的 $\pi-\pi$ 相互作用. 研究发现, 芳香螺旋 $\mathbf{A H}$ 的内部中空结构 对碱金属离子具有选择性识别的特性, 其选择性顺序 为: $\mathrm{Rb}^{+}>\mathrm{K}^{+}>\mathrm{Cs}^{+}>\mathrm{Na}^{+}>\mathrm{Li}^{+}$. 由于芳香螺旋 $\mathbf{A H}$ 的内

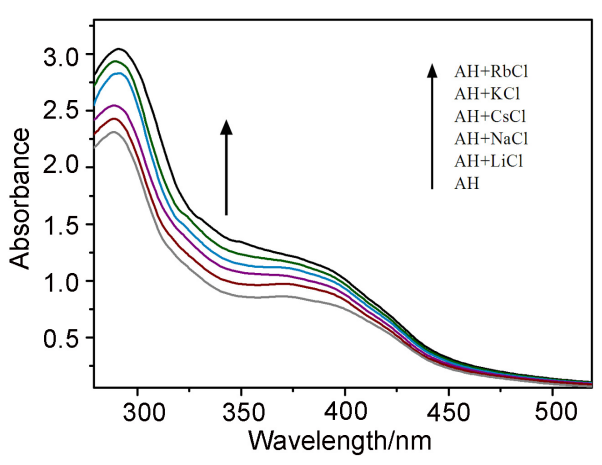

图 2 在 $400 \mu \mathrm{L} 25.5 \mu \mathrm{mol} / \mathrm{L}$ 的芳香螺旋 $\mathbf{A H}$ 的 DMSO 溶液中 加入 $200 \mu \mathrm{L} 1.05 \mathrm{mmol} / \mathrm{L}$ 的氯化碱金属盐水溶液后的紫外光 谱图

Figure 2 UV-Vis spectra of $\mathbf{A H}(400 \mu \mathrm{L}, 25.5 \mu \mathrm{mol} / \mathrm{L})$ in DMSO under different metal ion $(200 \mu \mathrm{L}, 1.05 \mathrm{mmol} / \mathrm{L})$ in $\mathrm{H}_{2} \mathrm{O}$

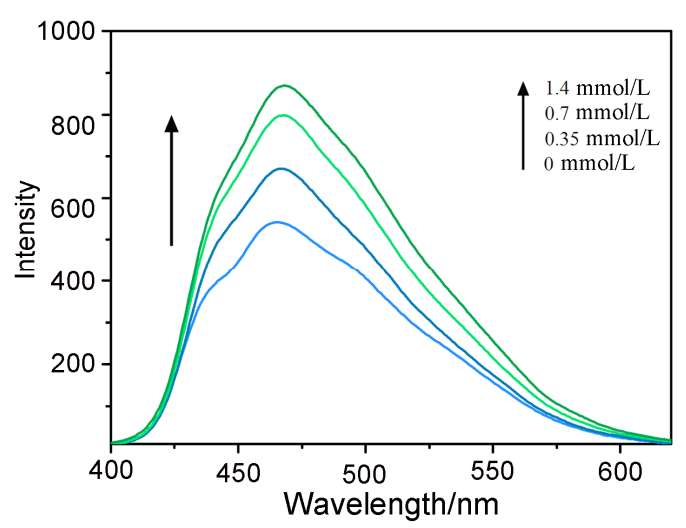

图 3 在 $400 \mu \mathrm{L} 25.5 \mu \mathrm{mol} / \mathrm{L}$ 的芳香螺旋 $\mathbf{A H}$ 的 DMSO 溶液中 加入 $200 \mu \mathrm{L}$ 不同浓度 $(1.05,2.1,4.2 \mathrm{mmol} / \mathrm{L})$ 的 $\mathrm{RbCl}$ 水溶液后 的苂光光谱图

Figure 3 Fluorescence spectra of $\mathbf{A H}(400 \mu \mathrm{L}, 25.5 \mu \mathrm{mol} / \mathrm{L})$ in DMSO under $\mathrm{RbCl}$ in $\mathrm{H}_{2} \mathrm{O}$ with different concentration $(200 \mu \mathrm{L}$, $1.05,2.1,4.2 \mathrm{mmol} / \mathrm{L}$ )

部结构环境以及其空腔尺寸, 它们对碱金属离子的识别 强弱实际上与它们的空间匹配程度相关. 本文用相对简 单的芳香螺旋 $\mathrm{AH}$ 成功地模拟了天然离子通道的结构与 性能，接下来将通过有机合成方法制备尺寸相当(通道 长度约 $3.5 \mathrm{~nm}$ ) 的单分子型离子通道, 研究此类离子通 道在磷脂膜乃至细胞膜上实现跨膜通道的工作. 这将对 深入研究离子通道相关疾病 ${ }^{[16]}$ 并发现特异性治疗药物 具有十分重要的理论和实际科学意义, 也将为从根本上 理解天然通道分子的作用机制打下重要基础.

\section{3 实验部分}

\section{1 仪器与试剂}

Bruker AVANCEIII 500MHz 核磁共振仪, 美国 PE Lambda-800 紫外可见近分光光度计, 日本岛津 5301PC 苂光光谱仪. 2,6-二氨基甲苯、偶氮二甲酸二异丙酯、三 
苯基磷、二苯醚、 $\mathrm{Pd} / \mathrm{C}$ 、甲酸铵、偏钒酸铵、PyBOP、 三氟乙酸均来自阿拉丁试剂网, 异丁醇购买于梯希爱 (上海)化成工业发展有限公司, 其他盐类均来自于国药 集团，所用试剂都为分析纯纯度，来自于北京化工厂， 无水 $N, N$-二甲基甲酰胺(DMF) 是由普通 DMF 与氢化钙 长期搅拌后减压蒸馏而来, 无水四氢呋喃和三乙胺都是 由其普通试剂与钠回流常压蒸馏得到的.

\section{2 实验方法}

3.2.1 4,6'-二异丁基-2,8'-二羧酸-10"- 甲基吡啶并 〔3,2-g〕喹啉(1)的合成

单体化合物 1 的合成方法参考文献[17]. 利用 2,6二氨基甲苯与丁炔二酸二甲酯加成, 加热环化形成单体 $\mathbf{b}$, 然后利用 Mitsunobu $^{[18]}$ 反应引入侧链异丁基得到单 体 $\mathbf{c}$, 最后通过水解得到 4,6'-二异丁基-2,8'-二羧酸-10"甲基吡啶并 (3,2-g) 喹啉.

\section{2 .2 化合物 $\mathbf{2}$ 的合成}

将充分干燥的 $1 \mathrm{~g}(2.3 \mathrm{mmol})$ 化合物 $1 、 1.1 \mathrm{~g}(2.1$ mmol) PyBOP、 $1.5 \mathrm{~mL}(1.1 \mathrm{mmol})$ 干燥三乙胺在 $10 \mathrm{~mL}$ 干燥 DMF 中分散, 反复抽真空通氮气 3 5 次. 最后在 氮气保护和避光条件下, 向上述体系中缓慢滴加 $1.1 \mathrm{~mL}$ $(1.1 \mathrm{mmol}) 1.0 \mathrm{~mol} / \mathrm{L}$ 的肼的四氢呋喃溶液. 混合反应体 系后室温摚拌 $1 \mathrm{~h}, 80{ }^{\circ} \mathrm{C}$ 恒温摚拌 $20 \mathrm{~h}$. 薄层色谱法监 测反应结束后, 减压蒸干有机溶剂, 固体残渣在无水甲 醇中分散, 过滤并用甲醇充分洗涤滤饼. 将所得固体在 $50{ }^{\circ} \mathrm{C}$ 真空干燥箱中充分干燥得 $0.85 \mathrm{~g}$ 红褐色固体, 产 率 90\%. m.p. $260 \sim 280{ }^{\circ} \mathrm{C}$; ${ }^{1} \mathrm{HNMR}\left(500 \mathrm{MHz}, \mathrm{CDCl}_{3}\right.$ / $1 \%$ TFA) $\delta: 11.35 \sim 11.63(\mathrm{~m}, \mathrm{NH}) 9.51 \sim 9.91(\mathrm{~m}, \mathrm{Ar}-\mathrm{H})$, $7.66 \sim 8.42(\mathrm{~m}, \mathrm{Ar}-\mathrm{H}), 3.94 \sim 4.49\left(\mathrm{~m}, \mathrm{OCH}_{2}\right), 2.92 \sim 3.52$ (m, Ar- $\left.\mathrm{CH}_{3}\right), 2.4 \sim 2.62(\mathrm{~m}, \mathrm{CH}), 1.01 \sim 1.38\left(\mathrm{~m}, \mathrm{CH}_{3}\right)$; IR (KBr) $v$ : 3284, 3068, 2962, 2926, 2871, 1714, 1677, 1604, $1555,1496,1454,1392,1369,1300,1253,1207,1173$, 1154, 1142, 1107, 1032, 892, 868, 837, 790, 774, 712 $\mathrm{cm}^{-1}$; GPC: $M_{\mathrm{n}}=3015$, PDI: 1.21. Anal. calcd for $\left[\mathrm{C}_{23} \mathrm{H}_{26} \mathrm{~N}_{4} \mathrm{O}_{4}\right]_{9}$ : C 65.39, H 6.20, N 13.26; found C 65.26, $\mathrm{H} 6.14, \mathrm{~N} 12.75$.

\subsection{2 芳香螺旋 $\mathbf{A H}$ 的合成}

在氮气保护条件下, 将 $0.85 \mathrm{~g}$ 化合物 2 在三氯氧磷 中 $105{ }^{\circ} \mathrm{C}$ 煮沸 $5 \mathrm{~h}$. 反应结束, 减压抽干三氯氧磷. 剩 余固体用三氯甲烷溶解并用饱和碳酸氢钠溶液中和之 后, 依次用蒸馏水、乙酸乙酯、无水甲醇充分洗涤. 最 后过滤收集, 得黑褐色固体, 产率 76\%. m.p. 280 $300{ }^{\circ} \mathrm{C} ;{ }^{1} \mathrm{H}$ NMR $\left(500 \mathrm{MHz}, \mathrm{CDCl}_{3} / 1 \% \mathrm{TFA}\right) \delta$ : 9.37 $9.82(\mathrm{~m}$, Ar-H), $7.61 \sim 8.27(\mathrm{~m}$, Ar- $\mathrm{H}), 4.0 \sim 4.78(\mathrm{~m}$, $\left.\mathrm{OCH}_{2}\right), 2.88 \sim 3.68\left(\mathrm{~m}, \mathrm{Ar}-\mathrm{CH}_{3}\right), 2.2 \sim 2.70(\mathrm{~m}, \mathrm{CH})$, $0.95 \sim 1.46\left(\mathrm{~m}, \mathrm{CH}_{3}\right)$; IR (KBr) v: 3090, 2960, 2930, 2873, 1714, 1667, 1604, 1555, 1482, 1465, 1392, 1369, 1300, 1273, 1207, 1173, 1154, 114 2, 1107, 1032, 970, 948, 892, $868,837,790,774,757,688 \mathrm{~cm}^{-1}$; GPC: $M_{\mathrm{n}}=2900$, PDI: 1.18. Anal. calcd for $\left[\mathrm{C}_{23} \mathrm{H}_{24} \mathrm{~N}_{4} \mathrm{O}_{3}\right]_{7}: \mathrm{C} 68.23, \mathrm{H} \mathrm{5.93,} \mathrm{N}$ 13.84; found C 67.97, H5.96, N 13.30.

辅助材料(Supporting Information) ${ }^{1} \mathrm{H}$ NMR, GPC, IR 谱图. 这些材料可以免费从本刊网站(http://sioc-journal. $\mathrm{cn} /$ )上下载.

\section{References}

[1] Hille, B. Ionic Channels of Excitable Membranes, 3rd ed., Sinauer Associates: Sunderland, MA, 2001.

[2] Tabushi, I.; Kuroda, Y.; Yokota, K. Tetrahedron Lett. 1982, 23(44), 4601.

[3] Cornell, B. A.; Braach-Maksvytis, V.; King, L.; Osman, P.; Raguse, B.; Wieczorek, L.; Pace, R. Nature 1997, 387(6633), 580.

[4] Das, G.; Talukdar, P.; Matile, S. Science 2002, 298(5598), 1600.

[5] Sakai, N.; Matile, S. Langmuir 2013, 29, 9031.

[6] Zhang, D.; Yang, Z.Y. J. Phys. Chem. B 2011, 115(49), 4333.

[7] Blake, A. J.; Cooke, P. A.; Doyle, K. J.; Gair, S.; Simpkins, N. S. Tetrahedron Lett. 1998, 39, 9093.

[8] Goto, H.; Katagiri, H.; Furusho, Y.; Yashima, E. J. Am. Chem. Soc. 2006, 128,7176 .

[9] Nakano, T.; Okamoto ,Y. Chem. Rev. 2001, 101, 4013.

[10] de Witte, P. A. J.; Castriciano, M.; Cornelissen, J .J. L. M.; Scolaro, L. M.; Nolte, R. J. M.; Rowan, A. E. Chem. Eur. J. 2003, 9, 1775.

[11] Delsuc, N; Godde, F.; Kauffmann, B.; Léger, J. M.; Huc, I. J. Am. Chem. Soc. 2007, 129, 11348.

[12] Kudo, M.; Maurizot, V.; Kauffmann, B.; Tanatani, A.; Huc, I. J. Am. Chem. Soc. 2013, 135, 9628.

[13] Chandramouli, N.; Ferrand, Y.; Lautrette, G.; Kauffmann, B.; Mackereth, C. D.; Laguerre, M.; Dubreuil, D.; Huc, I. Nat. Chem. 2015, 7, 334.

[14] Zhu, J. Y.; Dong, Z. Y.; Lei, S. B.; Cao, L. L.; Yang, B.; Li, W. F.; Zhang, Y. C.; Liu, J. Q. ; Shen, J. C. Angew. Chem., Int. Ed. 2015, $54,3097$.

[15] Ren, C. L.; Victor, M.; Zhao, H. Q.; Shen, J.; Zhou, F.; Ong, W. Q.; Du, Z. Y.; Zhang, K.; Su, H. B.; Zeng, H. Q. J. Am. Chem. Soc. 2011, 133, 13930.

[16] Zhang, Z. M.; Qiu, F. Z. World Chin. J. Digestol. 2005, 13(5), 585 (in Chinese). (张宗明, 表法祖, 世界华人消化杂志, 2005, 13(5), 585.)

[17] Berni, E.; Kauffmann, B.; Bao, C. Y.; Lefeuvre, J.; Bassani, D. M.; Huc, I. Chem. Eur. J. 2007, 13, 8463.

[18] Ren, X. F.; Xu, J. L.; Chen, S. H. Chin. J. Org. Chem. 2006, 26, 454 (in Chinese).

(任新锋, 徐菁利, 陈思浩, 有机化学, 2006, 26, 454.) 